\title{
Aproximación a la salud y bienestar en estudiantes universitarios: importancia de las variables sociodemográficas, académicas y conductuales ${ }^{1}$
}

\author{
Mónica Cassaretto Bardales ${ }^{2}$, Patricia Martínez Uribe ${ }^{3}$, Mariela Tavera Palomino ${ }^{4}$ \\ Pontificia Universidad Católica del Perú
}

El presente estudio pretende aproximarse a la salud y bienestar en un grupo de estudiantes de una universitaria privada de Lima, Perú. Para ello, describe aspectos asociados a las mismas y analiza de qué manera variables sociodemográficas, académicas y conductuales inciden en ella. El estudio contó con 1073 estudiantes y se encontró que presentan una percepción levemente positiva de su salud pero con importante presencia de sintomatología física y psicológica. Se realizaron análisis de regresión múltiple para cada indicador de salud, hallándose modelos pequeños pero robustos para Salud Percibida Global $\left(R^{2}=.16, p<.001\right)$ Salud Física $\left(R^{2}=.09, p<.001\right)$ y Salud Mental $\left(R^{2}=.15, p<.001\right)$. Respecto al bienestar, se encontraron modelos para Afecto Positivo $\left(R^{2}=.16, p<.001\right)$, Afecto Negativo $\left(R^{2}=.15, p<\right.$ $.001)$ y Florecimiento $\left(R^{2}=.19, p<.001\right)$. Se espera que los resultados ayuden a impulsar iniciativas para la promoción de la salud en la universidad.

Palabras clave: salud, bienestar, florecimiento, estudiantes universitarios, promoción de la salud

Health and Well-being in university students: the importance of sociodemographic, academic and behavioral variables

The aims of the study were to evaluate health and well-being among a sample of Peruvian students attending a private university and identify sociodemographic, academic and behavioral variables associated with them. The study assessed 1073 students; the majority reported a positive perception of their health but there is evidence of physical and psycho-

1 Investigación financiada por la Pontificia Universidad Católica del Perú a través de la Dirección de Gestión de la Investigación (DGI).

2 Doctora en Psicología. Docente asociada del departamento de Psicología de la Pontificia Universidad Católica del Perú. Coordinadora del Grupo de Investigación en Psicología y Salud: Entornos Saludables (PyS:Es). Dirección postal: Av. Universitaria 1801, Lima. Contacto: mcassar@pucp.edu.pe. https://orcid.org/0000-0002-4880-6092

3 Doctora en Psicología de la Universidad Autónoma de Barcelona. Profesora principal del Departamento de Psicología. Miembro del Grupo de Investigación en Psicología y Salud: Entornos Saludables (PyS:Es). Dirección postal: Av. Universitaria 1801, Lima. Contacto: pmartin@pucp.pe. https://orcid.org/0000-0001-8361-227X

4 Magíster en Psicología Clínica de la Salud. Consultora nacional e internacional en Proyectos en Prevención y Promoción de la Salud. Docente por horas del departamento de Psicología de la Pontificia Universidad Católica del Perú. Miembro del Grupo de Investigación en Psicología y Salud: Entornos Saludables (PyS:Es). Dirección postal: Av. Universitaria 1801, Lima. Contacto: mariela.tavera@pucp.pe. https://orcid.org/0000-0002-1502-8159 
logical symptoms. Multiple regression analyses were performed for each health indicator, and there were small but robust models for Global Perceived Health $\left(R^{2}=.16, p<.001\right)$ Physical Health $\left(R^{2}=.09, p<.001\right)$ and Mental Health $\left(R^{2}=.15, p<.001\right)$. Regarding wellbeing, models were found for Positive Affect $\left(R^{2}=.16, p<.001\right)$, Negative Affect $\left(R^{2}=.15\right.$, $p<.001)$ and Flourishing $\left(R^{2}=.19, p<.001\right)$. These findings support the need for health promotion initiatives in universities.

Keywords: Health, wellbeing, flourishing, university students, health promotion

\begin{abstract}
Abordagem à saúde e ao bem-estar em universitários: importância das variáveis sóciodemográficas, acadêmicas e comportamentais

Este estudo tem como objetivo se aproximar à saúde e ao bem-estar em um grupo de estudantes de uma universidade privada em Lima, Peru. Para isso, descrevessem aspectos associados e analisa como as variáveis sociodemográficas, acadêmicas e comportamentais os afetam. Participaram d estudo 1073 estudantes e descobriu-se que eles tinham uma percepção ligeiramente positiva de sua saúde, mas com uma presença significativa de sintomas físicos e psicológicos. Análises de regressão múltipla foram realizadas para cada indicador de saúde, encontrando modelos pequenos mas robustos, para a Saúde Geral Percebida $\left(R^{2}=.16\right.$, $p<.001)$ Saúde Física $\left(R^{2}=.09, p<.001\right)$ e Saúde Mental $\left(R^{2}=.15, p<.001\right)$. Em relação ao bem-estar, foram encontrados modelos para Afeto Positivo $\left(R^{2}=.16, p<.001\right)$, Afeto Negativo $\left(R^{2}=.15, p<.001\right)$ e Florescimento $\left(R^{2}=.19, p<.001\right)$. Os resultados devem ajudar a impulsionar as iniciativas de promoção da saúde na universidade.

Palavras-chave: saúde, bem-estar, florescimento, estudantes universitários, promoção da saúde
\end{abstract}

Approche de la santé et du bien-être chez les étudiants universitaires : importance des variables sociodémographiques, académiques et comportementales

L'objectif de l'étude était d'analyser la situation en matière de santé et de bien-être dans un groupe d'étudiants d'une université privée péruvienne et d'analyser comment les variables sociodémographiques, académiques et comportementales l'affectent. L'étude avait un échantillon de 1073 étudiants. Il a été constaté que dans la majorité une perception positive de leur santé prévalait mais avec la présence de symptômes physiques et psychologiques. Des analyses de régression multiples ont été effectuées pour chaque indicateur de santé, trouvant des modèles pour la santé générale perçue $\left(R^{2}=0,16, \mathrm{p}<.001\right)$, la santé physique $\left(R^{2}=.09, p<.001\right)$ et la santé mentale $\left(R^{2}=.15, p<.001\right)$. Concernant le bien-être, des modèles d'affect positifs $\left(R^{2}=.16, \mathrm{p}<.001\right)$, d'affect négatifs $\left(R^{2}=.15, p<.001\right)$ et l'épanouissement $\left(R^{2}=.19, p<.001\right)$ ont été trouvés. Les résultats devraient aider à stimuler les initiatives de promotion de la santé à l'université.

Mots-clés: santé, bien-être, épanouissement, étudiants universitaires, promotion de la santé 
La salud constituye en la actualidad una de las mayores preocupaciones de los individuos pero también de los gobiernos de cada país. La salud es definida como un estado de completo bienestar físico, mental y social y, no meramente la ausencia de dolencias o enfermedad (Organización Mundial de la Salud OMS, 1948). Esta definición implica que la enfermedad es vista como producto de la ruptura del equilibrio de uno o más de estos niveles mientras que, la salud reflejaría el mantenimiento del equilibrio (Amigo et al., 2003; Taylor, 2007).

Desde una visión más moderna, se considera que la salud puede ser evaluada tomando en cuenta distintos indicadores, entre ellos, el autorreporte del sujeto sobre su propia salud, así como su funcionamiento en distintas áreas de la vida, la presencia de patología y molestias, así como lo aspectos vinculados al bienestar y estilo de vida (Chandola $\&$ Jenkinson, 2000; Idler \& Benyamin; 1997; Pinquart, 2001).

Por ello, la OMS plantea que el estudio de la salud si bien puede dar evidencia de la presencia de sintomatología física y/o mental no debe descuidar el lado positivo de la vida, siendo el objetivo principal del trabajo en salud el logro de mayores niveles de bienestar, además de encontrar aquellas condiciones necesarias que la promuevan. El bienestar puede definirse como el estado de la persona en el que se le hace sensible el buen funcionamiento somático y psíquico que es percibido por el propio individuo (RAE, 2001), este concepto está ligado un funcionamiento óptimo y no solo la ausencia de enfermedad mental (Ryan $\&$ Deci, 2001).

La salud no es un mero estado, es producto de un proceso de relaciones dinámicas y bidireccionales entre dimensiones y competencias individuales (biopsicosociales) y características ambientales (biofísicas, sanitarias, socio-económicas-culturales), cuyo resultado es un estado caracterizado por el equilibrio y el correspondiente bienestar biopsicosocial, siendo la enfermedad la pérdida momentánea o duradera, de 
dicho equilibrio dinámico. Bajo esa lógica, la OMS (2002) plantea que la relación entre el individuo y su contexto asume un rol determinante en la construcción de su salud y bienestar, el entorno en el que se desenvuelve el individuo es importante para el cuidado de la salud, y es allí donde se puede generar políticas que impulsen su promoción. Es por ello que, Lange y Vio (2006) señalan que los ambientes cotidianos donde la personas trabajan, aprenden, juegan o estudian son espacios idóneos para el desarrollo de propuestas de promoción de la salud.

En Latinoamérica, existen investigaciones que han empezado a analizar cómo es la salud dentro de algunos contextos como las universidades. Sin embargo, dichas investigaciones se han centrado en describir la salud percibida de forma global, la adaptación a la vida universitaria o han incorporado la recolección de indicadores aislados de sintomatología ansiosa o depresiva en los estudiantes (Barraza, 2008; Chau \& Saravia, 2014, 2016; Chau \& Vilela, 2017; Consorcio de Universidades, 2006; Feldman et al., 2008; Gonzáles-Ramírez et al., 2009). Dando un cuadro aún incompleto sobre otros aspectos vinculados a la salud que podrían estar siendo poco reconocidos.

A nivel mundial, estudios como los dirigidos por la American College Association (ACHA) con 80139 universitarios encontró que el $83 \%$ de los evaluados consideraba su salud entre buena a excelente; sin embargo, existía alta prevalencia de sintomatología física como alergias, asma, dolor de espalda, bronquitis, migrańas, presión alta, infección del tracto urinario, entre otros (ACHA, 2015). Otros estudios coinciden mostrando la presencia de quejas somáticas frecuentes tales como dolor de cabeza y espalda, falta de energía, somnolencia, dolores de estómago, síntomas de estreńimiento, movimientos intestinales, diarrea, náuseas, palpitaciones o taquicardias y sensación de falta de aire en esta población (El Ansari et al., 2011; El Ansari et al., 2013; El Ansari et al., 2014; Barraza, 2007, 2008; Feldman et al., 2008; González-Ramírez et al., 2009; Hussain et al., 2013).

En el Perú, un estudio con 11042 jóvenes entre 15 y 29 años peruanos muestra que el $55 \%$ de los mismos reporta que se ha sentido tenso, nervioso o inquieto; el $51 \%$ cansado y aburrido la mayor 
parte del tiempo; el $47.5 \%$ ha sufrido constantemente de dolores de cabeza, cuello o espalda; y el $44 \%$ ha estado triste y sin deseo por realizar sus actividades comunes y un 56.4\% haber sufrido cambios en el ritmo de sueño (Secretaría Nacional de la Juventud, SENAJU, 2012). En la misma línea, otras investigaciones señalan que un porcentaje importante de los universitarios podrían estar experimentando diversos cuadros de ansiedad y depresión (Agudelo et al., 2008; Barraza, 2003, 2007; Micin \& Bagladi, 2011; Pérez-Villalobos et al., 2011).

A partir de lo anterior, es necesario indagar de forma más amplia diversos indicadores de salud y bienestar en dicha población con el fin de generar propuestas adecuadas a sus reales necesidades; permitiéndonos de esta manera mejorar aquellas condiciones que la perjudican, además de potenciar aquellas otras que la protegen dentro de la universidad. El descuido del estudio de la misma puede llevar a que la universidad se convierta en un espacio donde se deteriora la calidad de vida de los individuos, y un espacio en el que se vayan desarrollando una serie de patologías físicas, emocionales y sociales; todas de alto costo personal como social. Además, si bien existe evidencia que apunta a señalar mayores niveles de salud en estudiantes varones (Becerra, 2013; Cornelisse-Vermaat et al., 2006; El Ansari \& Stock, 2016; El Ansari et al., 2011; Latas et al., 2014); es menos consistente los resultados sobre características como la facultad y ciclo de estudio, y menos la investigación en Latinoamérica sobre las variables asociadas conductuales como son el ejercicio y sueño.

Tomando en cuenta todo ello, es que se plantea un estudio cuyo objetivo es describir la salud de un grupo de estudiantes de una universidad privada de Lima, tomando en cuenta indicadores de salud percibida, bienestar, sintomatología, y estilo de vida, y analizar la relación entre salud y bienestar con variables sociodemográficas, académicas y conductuales. 


\section{Método}

\section{Participantes}

Este estudio contó con la participación de 1073 estudiantes pertenecientes a una universidad privada de Lima, Perú. Sus edades oscilaban los 16 y los 30 ańos $(M=19.97, D E=2.4)$. De este grupo el $55.9 \%$ fueron varones y $44.1 \%$ mujeres. El $17.4 \%$ de los estudiantes refieren que estudian y trabajan, y el $31.9 \%$ señalan tener pareja actual por un mínimo de 2 meses de relación. Respecto a condiciones académicas, los estudiantes provenían de 14 facultades de la universidad, el $65 \%$ se encontraba cursando carreras de ciencias y el 35\%, carreras de letras. Además, se tomó en cuenta ciclo en el que el alumno se encuentra matriculado y el nivel de estudio de la carrera; así, el $48 \%$ de los estudiantes se encuentra cursando los ciclos de inicio de carrera, $22 \%$ los de mitad y el $30 \%$ cursando los ciclos de final de la carrera. Cabe señalar que, el muestreo utilizado fue no probabilístico por cuotas (Dattalo, 2010), lo que permitió una distribución proporcionada entre el número de encuestados de acuerdo a la facultad y el nivel en la carrera (inicios, medios o final) en los que se encuentran. El criterio de inclusión incluyó estar matriculado en el semestre de estudio. Todos los estudiantes firmaron un consentimiento informado en el que se expresaba su intención de participar en esta investigación y el reconocimiento de sus derechos dentro de la misma.

\section{Medición}

Para la medición de la salud se utilizó el Cuestionario de Salud SF-36 (Ware \& Sherbourne, 1992) y las dos escalas de Bienestar (SPANE y Florecimiento) creadas por Diener et al. (2010) y una ficha de autoreporte.

El Cuestionario de Salud SF-36 desarrollado por Ware y Sherbourne en 1992 es una escala genérica que proporciona un perfil del estado de salud de las personas y es aplicable tanto a población clínica 
como población general (Ware \& Gandek, 1998; Ware, Snow, Kosinski y Gandek, 1993). Consta de 36 ítems que describen distintos indicadores de salud; entre ellos se encuentran la medida de salud percibida global, dos indicadores generales de salud (física y mental) y ocho áreas específicas. El SF-36 ha sido traducido a distintos idiomas como parte del proyecto "Internacional Quality of Life Assessment" (IQOLA); por ello, el instrumento ha sido adaptado en más de 40 países y existen más de 500 publicaciones con las versiones traducidas del inglés. Respecto a sus propiedades psicométricas, existen estudios llevados a cabo por el proyecto IQOLA que encuentran que la confiabilidad de la prueba excede el .80 para las ocho dimensiones y el .90 para los indicadores generales de salud. En estos estudios se confirma además la estructura factorial del instrumento con varianzas explicadas que superan el $76 \%$ (Ware \& Gandek, 1998).

En el Perú, Salazar y Bernabé (2012) han evaluado las propiedades psicométricas del instrumento en una muestra de 4344 personas entre 15 y 64 años provenientes de 4 ciudades del país. Los índices de consistencia interna alfa de Cronbach para las ocho dimensiones específicas se ubican en entre .66 y .92. Los autores realizaron asimismo, un análisis factorial confirmatorio que mostraron que un modelo de 8 factores de primer orden y 2 de segundo orden era la estructura factorial más adecuada ya que mostraba mejores índices de ajuste a los datos de $C F I=.96$ y $R M S E A=.05$ en comparación con modelos alternativos. En la presente investigación se hallaron índices de consistencia interna alfa de Cronbach de .85 para el área Salud física y de .86 para Salud mental. Las subescalas o dimensiones del SF-36 presentaron un alfa de Cronbach entre .75 a .86 .

Para evaluar el bienestar se utilizaron las dos escalas creadas por Diener et al. (2010) como parte de su modelo integrativo de bienestar: SPANE y Florecimiento. La afectividad se mide a través de las escalas SPANE, que evalúan afecto positivo y negativo en un amplio rango de emociones; cuenta con 12 ítems calificados en formato Likert, proporciona una puntuación para los sentimientos positivos (SPANE-P, 6 ítems) y una puntuación para los sentimientos negativos (SPANE-N, 
6 ítems). La escala de Florecimiento (FS) evalúa el crecimiento psicológico, sentido de vida, relaciones positivas y optimismo entre otros aspectos del desarrollo humano; cuenta con 8 ítems en formato Likert. Estas escalas han demostrado muy buenas propiedades psicométricas como lo demuestran los estudios de Diener et al. (2010) en Estados Unidos y Sigapur; Silva y Caetano en Portugal (2011); Howell y Buro (2014) en Canadá o Li et al. (2013) en China.

En Perú, Cassaretto y Martínez (2017), realizaron una investigación para estudiar las propiedades psicométricas de la versión en español de las escalas; para lo cual realizaron dos estudios. El primero, contó con la participación de 565 estudiantes de una universidad privada de Lima. En este, se encontró que las confiabilidades para las tres escalas eran adecuadas, obteniendo alfas de .89, .91 y .87, para las escalas FS, SPANE-P y SPANE-N, respectivamente. Además, analizando la estructura factorial de las escalas, coincidieron en la existencia de un solo factor para cada una de estas. En el segundo estudio, el cual contó con la participación de otros 201 estudiantes, también de una universidad privada de Lima, se encontraron alfas de .86, .85 y .79, para las escalas FS, SPANE-P y SPANE-N, respectivamente. Asimismo, este estudio halló evidencia de validez convergente y divergente de las escalas realizando correlaciones con distintos cuestionarios que midan aspectos asociados al bienestar. Asimismo, el análisis factorial confirmatorio usando el índice de ajuste corregido Satorra-Bentler arrojó índices adecuados para los modelos de florecimiento y afectividad; encontrándose, también, que el chi cuadrado $\left(\chi^{2}\right)$ de las escalas indicaba que la estructura factorial planteada inicialmente para las pruebas era adecuada. En este estudio se encontraron índices de consistencia interna de .90 para la escala SPANE-P, .85 para SPANE-N y .91 para la escala de Florecimiento.

La Ficha de Autorreporte: La cual recoge información sobre las características sociodemográficas (sexo, edad, lugar de nacimiento, condición laboral, estado de relación de pareja, nivel socioeconómico medido a partir de la escala de pago de la universidad, entre otros datos); académicos (ciclo de estudio, nivel de carrera, facultad, satisfacción con 
la carrera, satisfacción con el rendimiento) y otros indicadores de salud y conductas que son partes del estilo de vida de los universitarios como datos de talla y peso, reporte de presencia de problemáticas de salud en el último año, una lista de síntomas y/o problemas de salud aguda. Además. Se solicitó información sobre el sueño, regularidad de práctica de ejercicio físico y consumo de medicamentos.

\section{Procedimiento}

El proyecto de investigación contó con la aprobación de un comité de ética acreditado por la universidad. Se procedió a solicitar autorización a las autoridades de cada facultad al interior de la universidad, ni bien se contaba con la misma se procedió a establecer contacto con los profesores para permitirnos aproximarnos a sus aulas en una fecha previamente pactada. En ese contexto se invitaba a los estudiantes a participar del estudio firmando previamente un consentimiento informado en el cual se les indicaba los objetivos de la investigación y sus derechos como participantes (participación voluntaria, anonimato y el derecho a retirarse de la investigación en cualquier momento si así lo desearan). A los alumnos menores de edad se les entregó un asentimiento informado y una ficha informativa para los padres. Los instrumentos fueron administrados en el año 2016 antes de la 6ta semana de clases. La aplicación tuvo una duración promedio de 20 minutos.

\section{Análisis de datos}

Se utilizó el programa estadístico SPSS-21 para analizar los datos obtenidos de la muestra. Primero, se analizó la confiabilidad de los instrumentos empleados en la recopilación de datos de la muestra, utilizando el cálculo de alfa de Cronbach para establecer si eran consistentes los resultados. A continuación, se realizaron la prueba de normalidad Kolmogorov-Smirnov, se estudió el perfil de asimetría y curtosis de las pruebas y en función de los resultados se procedió a trabajar con esta- 
dísticos paramétricos (Kline, 2010). Luego, se realizaron los análisis descriptivos de las distintas características de los participantes y de los indicadores de salud y bienestar. Posteriormente, se realizaron contrastes de medias y correlaciones a través de los estadísticos t-Student y el coeficiente $r$ de Pearson. Finalmente, para estudiar la relación que las variables de salud (global, física y mental) y bienestar (afecto positivo, afecto negativo y florecimiento) tenían con otras variables sociodemográficas o académicas se realizaron análisis de regresión lineal múltiple para cada componente.

\section{Resultados}

Respecto a la percepción de salud global (figura 1), el $81 \%$ de los evaluados reportan una percepción positiva o favorable. De manera específica un $49.6 \%$ la califican como buena, 28,.5\% como muy buena y $3.1 \%$ como excelente. En el polo menos favorable, se encuentra la opción regular (17.4\%) y solo un $1.4 \%$ de los participantes califican su salud como mala.

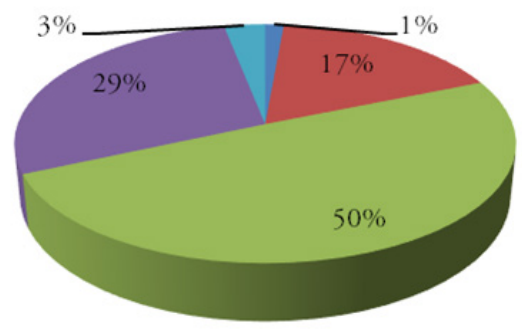

a Mala $\square$ Regular $\square$ Buena $\square$ Muybuena Excelente

Figura 1. Percepción general de la salud

En la Tabla 1 se muestran los descriptivos de cada componente y dimensión del SF-36. Se encuentran que existen diferencias significativas entre los niveles de salud física con los de salud mental. Se encontró 
que en la muestra la salud física es significativamente mayor a la salud mental $[t(1062)=17.59 ; p \leq .001 ; d=0.54)]$. Se encuentra que, el área de salud mejor preservada en el grupo de participantes es la Función Fisica, lo cual implica que los estudiantes no sufren problemas de salud que interfieran considerablemente en sus actividades físicas de la vida diaria como lo son las de cuidado personal, caminar, o subir escaleras. Por otro lado, las áreas de salud más debilitadas de los alumnos son las de Vitalidad y Rol Emocional, indicando que la sensación de energía y vitalidades es el aspecto de la salud más afectado de los alumnos, seguido del grado de limitaciones que presentan sus problemas de salud emocional al trabajo y demás actividades cotidianas.

\section{Tabla 1}

Descriptivos del SF-36 en los estudiantes

\begin{tabular}{lcccc}
\hline & Mínimo & Máximo & Media & $\begin{array}{c}\text { Desviación } \\
\text { estándar }\end{array}$ \\
\hline Función Física & 40 & 100 & 89.95 & 12.07 \\
Rol Físico & 0 & 100 & 62.17 & 39.26 \\
Rol Emocional & 0 & 100 & 56.27 & 43.7 \\
Vitalidad & 5 & 100 & 53.02 & 17.57 \\
Salud Mental & 8 & 96 & 62.18 & 19.06 \\
Función Social & 0 & 100 & 68.48 & 23.37 \\
Dolor Físico & 0 & 100 & 67.02 & 20.96 \\
Salud General & 10 & 100 & 59.84 & 17.91 \\
\hline Componente Salud Física & 30 & 98.75 & 69.71 & 16.83 \\
Componente Salud Mental & 13.25 & 98 & 59.86 & 20.92 \\
\hline
\end{tabular}

Por otro lado, el 94\% de los participantes reportó haber tenido por lo menos una dolencia o molestia de salud en el último ańo; el número promedio de dolencias reportado por los estudiantes fue 4.79 $(D E=2.56)$. En la figura 2 se observan las condiciones médicas de mayor frecuencia son mayormente dolor sea en el cuello, hombros, 
espalda o cabeza $(85.84 \%)$, ansiedad $(58.3 \%)$, problemas estomacales (46.7\%), somnolencia $(45.3 \%)$, fatiga $(42.8 \%)$, depresión $(30.4 \%)$ alergias $(29.1 \%)$, insomnio $(24.9 \%)$ y problemas respiratorios $(23.3 \%)$.

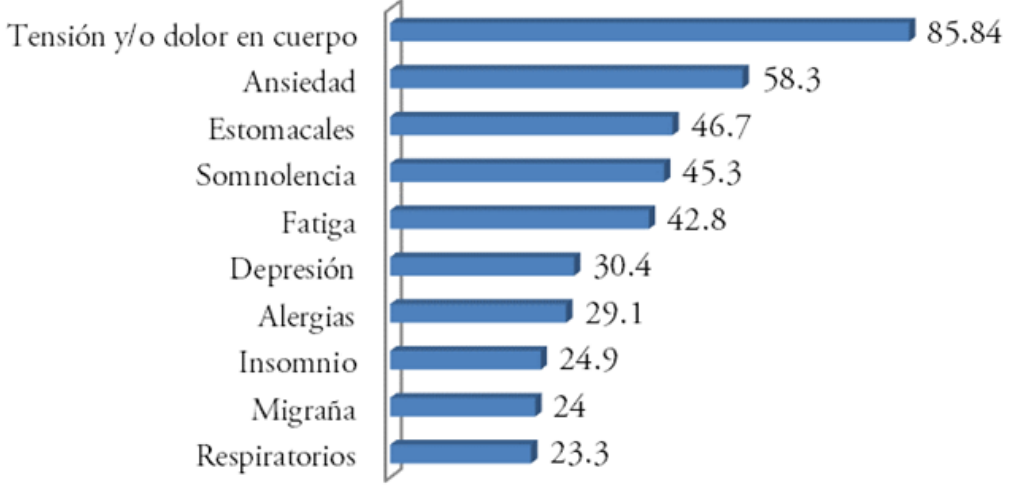

Figura 2. Porcentaje de alumnos que reportan tipo de dolencia en el último año

Respecto al índice de masa corporal (IMC) reportado, el cual se obtiene usando la fórmula de Quetelet, se halla un peso mayormente adecuado $(X=23.04$ y D.E. $=3.05)$. Tomando en cuenta las categorías clasificatorias del estado nutricional establecidas por la OMS puede observarse que la mayoría de los participantes $(71.5 \%)$ reportan un peso adecuado y un porcentaje menor (28.5\%) reportan uno inadecuado. Específicamente, $4.2 \%$ reporten bajo peso y 22\% sobrepeso.

Respecto a la percepción de la relación de alumno con la universidad, se indagaron sobre el nivel de motivación percibida a la carrera y se halló que esta es mayormente satisfactoria (45.4\%), muy satisfactoria $(25.3 \%)$, regular $(23 \%)$ y entre insatisfecho a muy insatisfactoria (6.2\%). Sobre la percepción de satisfacción con su rendimiento, esta es mayormente regular (45.4\%), seguida de satisfactorio (29.2), insatisfactorio (16.6\%), muy insatisfactorio (5.6\%) y muy satisfactorio $(3.2 \%)$. 
A nivel de las conductas de salud, encontramos que $27.1 \%$ de los evaluados indican que nunca practican ejercicio físico y/o deporte, $36.4 \%$ practican algo, 23\% regular, $10.7 \%$ bastante y 2.7 mucho. En términos del sueño, el $41 \%$ de los encuestados refiere un número de horas dentro de las recomendadas por la OMS para un sueño adecuado de acuerdo a la edad $(3.7 \%$ duerme menos de 5 horas diarias en promedio, $54.1 \%$ entre 5 a 6.5 horas, $41 \%$ entre 7 a 9 horas y $1.2 \%$ más de 9 horas). Tan solo un $1.1 \%$ reportar usar medicamentos para el sueño, consumen al menos alguna vez antidepresivos $8.4 \%$, ansiolíticos $6.6 \%$ y $51.2 \%$ analgésicos, cabe señalar que un $10.4 \%$ señala un consumo muy frecuentes de estos últimos.

En la Tabla 2 se presentan las diferencias entre los indicadores de salud y bienestar con variables sociodemográficas, académicas y conductuales se encontraron diferencias estadísticamente significativas a nivel de varias de las escalas de acuerdo a sexo del participante. Así, se encuentra que los varones, presentan mayores niveles de salud percibida global, salud mental, salud física y menores niveles de afecto negativo en comparación con las mujeres. Si bien no existieron diferencias respecto a las facultades de estudio, cuando estás se las agrupaban en carreras de letras o ciencias se encontró que los estudiantes de ciencias reportan mayores niveles de salud percibida global, salud mental, salud física y menores niveles de afecto negativo en comparación a sus pares de letras. También se halló que aquellos estudiantes que reportan que trabajan presentan menores niveles de afecto negativo mientras que, los que reportan tener pareja muestran mayores niveles de afecto positivo y florecimiento en comparación a quienes no lo tienen. Sobre el sueño, los que reportan un número de horas de sueño dentro de los rangos recomendados por la OMS (entre 7 a 9 horas) reportan mejor salud en los tres indicadores y menor afecto negativo que los que no cumplen esta pauta. Cabe seńalar que todas las diferencias fueron de tamańo pequeño de acuerdo al criterio de Cohen. 


\section{Tabla 2}

Diferencias en salud y bienestar de acuerdo a variables sociodemográficas, académicas y conductuales

\begin{tabular}{|c|c|c|c|c|c|c|c|}
\hline & \multicolumn{2}{|c|}{$\begin{array}{c}\text { Hombres } \\
(n=599-600)\end{array}$} & \multicolumn{2}{|c|}{$\begin{array}{c}\text { Mujeres } \\
(\mathrm{n}=457-461)\end{array}$} & \multirow{2}{*}{$t(g l)$} & \multirow{2}{*}{$p$} & \multirow{2}{*}{$d$} \\
\hline & $M$ & $D E$ & $M$ & $D E$ & & & \\
\hline Salud percibida & 56.71 & 19.48 & 49.67 & 19.31 & $5.85(986.13)$ & .000 & 0.36 \\
\hline Componente Salud Física & 72.96 & 16.36 & 67.94 & 17.13 & $4.84(1055)$ & .000 & 0.30 \\
\hline Componente Salud Mental & 63.19 & 19.86 & 57.28 & 19.63 & $4.81(1055)$ & .000 & 0.30 \\
\hline \multirow[t]{3}{*}{ Afecto Negativo } & 13.74 & 4.38 & 14.61 & 4.50 & $-3.16(1057)$ & .002 & -0.20 \\
\hline & \multicolumn{2}{|c|}{$\begin{array}{c}\text { Letras } \\
(\mathrm{n}=368)\end{array}$} & \multicolumn{2}{|c|}{$\begin{array}{l}\text { Ciencias } \\
(n=684)\end{array}$} & \multirow[t]{2}{*}{$t(g l)$} & \multirow{2}{*}{$p$} & \multirow[t]{2}{*}{$d$} \\
\hline & $M$ & $D E$ & $M$ & $D E$ & & & \\
\hline Salud percibida global & 50.88 & 20.324 & 55.11 & 19.195 & $-3.34(1056)$ & 0.001 & -0.21 \\
\hline Componente Salud Física & 68.04 & 18.028 & 72.25 & 16.100 & $-3.75(680.06)$ & 0.000 & -0.25 \\
\hline Componente Salud Mental & 57.47 & 20.123 & 62.37 & 19.665 & $-3.84(1056)$ & 0.000 & -0.25 \\
\hline \multirow[t]{3}{*}{ Afecto Negativo } & 14.81 & 4.580 & 13.76 & 4.341 & $3.69(1058)$ & 0.000 & 0.24 \\
\hline & \multicolumn{2}{|c|}{$\begin{array}{l}\text { Horas sueño } \\
\text { adecuado } \\
(n=437)\end{array}$} & \multicolumn{2}{|c|}{$\begin{array}{l}\text { Horas sueño } \\
\text { inadecuado } \\
(n=628)\end{array}$} & \multirow[t]{2}{*}{$t(g l)$} & \multirow[t]{2}{*}{$p$} & \multirow[t]{2}{*}{$d$} \\
\hline & $M$ & $D E$ & $M$ & $D E$ & & & \\
\hline Salud percibida global & 56.93 & 19.718 & 51.29 & 19.202 & $4.62(913.66)$ & 0.000 & 0.29 \\
\hline Componente Salud Física & 73.68 & 16.022 & 68.76 & 17.124 & $4.77(966.87)$ & 0.000 & 0.30 \\
\hline Componente Salud Mental & 63.35 & 19.854 & 58.94 & 19.835 & $3.55(1053)$ & 0.000 & 0.22 \\
\hline \multirow[t]{3}{*}{ Afecto Negativo } & 13.53 & 4.148 & 14.49 & 4.577 & $-3.57(987.50)$ & 0.000 & -0.22 \\
\hline & \multicolumn{2}{|c|}{$\begin{array}{l}\text { Tiene pareja } \\
\qquad(\mathrm{n}=328)\end{array}$} & \multicolumn{2}{|c|}{$\begin{array}{l}\text { No tiene pareja } \\
\text { Actual }(n=622)\end{array}$} & \multirow[t]{2}{*}{$t(g l)$} & \multirow{2}{*}{$p$} & \multirow[t]{2}{*}{$d$} \\
\hline & $M$ & $D E$ & $M$ & $D E$ & & & \\
\hline Afecto Positivo & 22.21 & 3.94 & 21.37 & 4.06 & $3.14(1036)$ & 0.002 & 0.21 \\
\hline \multirow[t]{2}{*}{ Florecimiento } & 44.90 & 7.80 & 42.89 & 8.22 & $3.75(1038)$ & 0.000 & 0.25 \\
\hline & \multicolumn{2}{|c|}{$\begin{array}{l}\text { Trabajan } \\
(\mathrm{n}=185)\end{array}$} & \multicolumn{2}{|c|}{$\begin{array}{l}\text { No trabajan } \\
\quad(n=874)\end{array}$} & $t(g l)$ & $p$ & $d$ \\
\hline
\end{tabular}

Afecto Negativo

$\begin{array}{llllllll}13.35 & 4.64 & 14.30 & 4.39 & -2.63(1057) & 0.009 & -0.21\end{array}$ 
La Tabla 3 muestra las correlaciones entre variables; así se encuentra relaciones entre todos los indicadores de salud y bienestar con la satisfacción con la carrera, satisfacción con el rendimiento y con la regularidad en la práctica del ejercicio físico. Los indicadores de salud y bienestar presentan relaciones de tamaño mediano a fuerte.

\section{Tabla 3}

Correlaciones entre indicadores de salud, bienestar, satisfacción carrera, rendimiento y regularidad del ejercicio

\begin{tabular}{lcccccccc}
\hline & 1 & 2 & 3 & 4 & 5 & 6 & 7 & 8 \\
\hline 1. Salud percibida global & $-\ldots$ & & & & & & & \\
2. Componente Salud física & $.51^{* *}$ & & & & & & & \\
3. Componente Salud mental & $.37^{* *}$ & $.50^{* *}$ & & & & & & \\
4. Afecto Positivo & $.35^{* *}$ & $.33^{* *}$ & $.58^{* *}$ & & & & & \\
5. Afecto negativo & $-.34^{* *}$ & $-.39^{* *}$ & $-.66^{* *}$ & $-.64^{* *}$ & & & & \\
6. Florecimiento & $.29^{* *}$ & $.28^{* *}$ & $.42^{* *}$ & $.60^{* *}-.49^{* *}$ & & & \\
7. Satisfacción carrera & $.11^{* *}$ & $.10^{* *}$ & $.22^{* *}$ & $.31^{* *}$ & $-.26^{* *}$ & $.35^{* *}$ & & \\
8. Satisfacción rendimiento & $.18^{* *}$ & $.14^{* *}$ & $.31^{* *}$ & $.30^{* *}$ & $-.27^{* *}$ & $.33^{* *}$ & $.36^{* *}$ & \\
9. Regularidad ejercicio & $.33^{* *}$ & $.19^{* *}$ & $.17^{* *}$ & $.20^{* *}-.17^{* *}$ & $.15^{* *}$ & 0.05 & $.10^{* *}$ \\
\hline
\end{tabular}

Nota: ${ }^{* *}$. La correlación es significativa en el nivel .01 (bilateral).

Posteriormente, se realizaron análisis de regresión lineal múltiple para estimar las variables que predicen salud (Tabla 4) y bienestar (Tabla 5 ) en los estudiantes. Respecto a los indicadores de salud, se encuentra que el sexo, tipo de carrera, grado de satisfacción con el rendimiento, tipo de sueño y frecuencia en la práctica del ejercicio físico predice un $17 \%$, 9\% y $15 \%$ de la varianza de la salud percibida global, componente salud física y del componente salud mental respectivamente (tabla 6). La satisfacción con la carrera no aportó significativamente a los modelos y fue retirado fueron retirados de los modelos finales.

Respecto al bienestar, se encuentra que tener pareja, satisfacción con la carrera, satisfacción con el rendimiento y frecuencia en la práctica del ejercicio físico predice un 17\% y $19 \%$ de la varianza del afecto 
positivo y florecimiento respectivamente. Para afecto negativo se halló un modelo con un poder de 15\%, siendo las variables significativas tipo de carrera, estar trabajando, grado de satisfacción con la carrera, satisfacción con el rendimiento, frecuencia en la práctica del ejercicio físico y tipo de sueño. Sexo del participante no aportó significativamente a los modelos y fue retirado fueron retirados de los modelos finales.

\section{Tabla 4}

Modelos de regresión múltiple para los indicadores de salud

\begin{tabular}{|c|c|c|c|c|c|c|c|c|c|}
\hline & \multicolumn{3}{|c|}{$\begin{array}{c}\text { Salud percibida } \\
\text { global }\end{array}$} & \multicolumn{3}{|c|}{$\begin{array}{c}\text { Componente } \\
\text { Salud física } \\
\end{array}$} & \multicolumn{3}{|c|}{$\begin{array}{l}\text { Componente } \\
\text { Salud mental }\end{array}$} \\
\hline & B & SE B & $\beta$ & B & SE B & $\beta$ & B & SE B & $\beta$ \\
\hline Sexo & -3.15 & 1.23 & $-0.08^{*}$ & -2.76 & 1.11 & $-0.08^{*}$ & -3.39 & 1.27 & $-0.08^{*}$ \\
\hline Tipo carrera & 4.35 & 1.26 & $0.11^{* * *}$ & 3.80 & 1.14 & $0.11^{* * *}$ & 5.38 & 1.30 & $0.13^{* * *}$ \\
\hline Sat. rendimiento & 3.59 & 0.63 & $0.17^{* * *}$ & 2.37 & 0.57 & $0.13^{* * *}$ & 6.97 & 0.65 & $0.31^{* * *}$ \\
\hline Ejercicio & 5.25 & 0.55 & & 2.43 & 0.49 & $0.15^{* * *}$ & 2.19 & 0.57 & $0.12^{* * *}$ \\
\hline Tipo sueño & 4.61 & 1.15 & $0.12^{* * *}$ & 4.29 & 1.03 & $0.12^{* * *}$ & 3.70 & 1.18 & $0.09^{* * *}$ \\
\hline $\mathrm{F}$ & \multicolumn{3}{|c|}{$40.30^{* * *}$} & \multicolumn{3}{|c|}{$19.86^{* * *}$} & \multicolumn{3}{|c|}{$37.62^{* * *}$} \\
\hline $\mathrm{R}^{2}$ ajustado & \multicolumn{3}{|c|}{.16} & \multicolumn{3}{|c|}{.09} & \multicolumn{3}{|c|}{.15} \\
\hline
\end{tabular}

Nota: ${ }^{*} p<.015,{ }^{* *} p<.01,{ }^{* * *} p<.001$.

\section{Tabla 5}

Modelos de regresión múltiple para los indicadores de bienestar

\begin{tabular}{|c|c|c|c|c|c|c|c|c|c|}
\hline & \multicolumn{3}{|c|}{ Afecto positivo } & \multicolumn{3}{|c|}{ Afecto negativo } & \multicolumn{3}{|c|}{ Florecimiento } \\
\hline & B & SE B & $\beta$ & B & SE B & $\beta$ & B & SE B & $\beta$ \\
\hline Pareja & -0.69 & 0.25 & $-0.08^{* *}$ & --- & -- & --- & -1.78 & 0.50 & $-3.59^{* * *}$ \\
\hline Tipo de carrera & --- & --- & --- & -1.26 & 0.27 & $-0.13^{* * *}$ & --- & --- & --- \\
\hline Trabajo & --- & -- & -- & 0.79 & 0.34 & $0.07^{*}$ & -.- & -.-- & -- \\
\hline Sat. carrera & 1.03 & 0.14 & $0.23^{* * *}$ & -0.94 & 0.15 & $-0.19^{* * *}$ & 2.31 & 0.28 & $8.41^{* * *}$ \\
\hline Sat. rendimiento & 0.88 & 0.14 & $0.20^{* * *}$ & -0.94 & 0.15 & $-0.19^{* * *}$ & 2.00 & 0.28 & $7.23^{* * *}$ \\
\hline Ejercicio & 0.69 & 0.11 & $0.18^{* * *}$ & -0.55 & 0.12 & $-0.13^{* * *}$ & 0.90 & 0.22 & $4.07^{* * *}$ \\
\hline Tipo de sueńo & --- & --- & --- & -1.05 & 0.26 & $-0.12^{* * *}$ & --- & --- & --- \\
\hline $\mathrm{F}$ & \multicolumn{3}{|c|}{$52.97^{* * *}$} & \multicolumn{3}{|c|}{$31.34^{* * *}$} & \multicolumn{3}{|c|}{$59.03^{* * *}$} \\
\hline $\mathrm{R}^{2}$ ajustado & \multicolumn{3}{|c|}{.17} & \multicolumn{3}{|c|}{.15} & \multicolumn{3}{|c|}{.19} \\
\hline
\end{tabular}




\section{Discusión}

La universidad es un espacio donde los adolescentes y jóvenes pasan la mayor parte de sus días, es un espacio donde estudian, aprenden, se relacionan y desarrollan nuevos hábitos que pueden permanecer para el resto de sus vidas. Dentro de este contexto se exponen a montos elevados de estrés, los cuales pueden afectar su salud, especialmente salud mental (Latas et al., 2014) y en tanto, existe evidencia de alta prevalencia de quejas somáticas y psicológicas (ACHA, 2015; El Ansari et al., 2011, 2014; Feldman et al., 2008; Gonzáles-Ramírez et al., 2009; Hussain et al., 2013) es que se considera necesario profundizar en el estudio de aquellos aspectos que afectan la salud y el bienestar en el universitario.

El presente estudio fue realizado con un grupo de estudiantes provenientes de una universidad privada de Lima, los diversos indicadores de salud muestran una situación compleja donde se alude a una percepción levemente positiva de la misma, especialmente en el área salud física sobre la salud mental; sin embargo, se reporta la presencia de algunos indicadores de sintomatología física y psicológica. Ante resultados similares, Becerra (2013) considera que los estudiantes no son plenamente conscientes de los riesgos que presentan respecto a su salud y por ello se pueden estar perpetuando respuestas que contribuyen a su deterioro. Es posible que los estudiantes no estén incorporando a su propia evaluación de la salud indicadores de quejas y problemas físicos y psicológicos, y en tanto, estas dificultades no califican aún como enfermedades serias o son claramente diagnosticadas sean minimizados. Esto se comprende si es que los estudiantes perciben su salud en términos de ausencia de enfermedad y no, como la OMS la plantea, como el estado de bienestar y equilibrio de las áreas biológicas, psicológicas y sociales.

En el presente estudio, existe un elevado número de alumnos que reportan la presencia de problemas o molestias en su salud en el último año, siendo las más señaladas la presencia de síndromes dolorosos, ansiedad, problemas estomacales y en el estado de ánimo. Estos 
resultados coinciden con reportes brindados por la ACHA (2015) en Estados Unidos, Pérez et al. (2010) en México o por SENAJU (2012) en el Perú. También, coinciden con los estudios de El Ansari et al. (2011) y El Ansari et al. (2013) sobre la presencia de quejas somáticas y psicológicas en universitarios del Reino Unido y de Egipto. Ello lleva a que posteriores análisis planteen que las problemáticas de salud de los universitarios giran alrededor de cuatro categorías: síntomas psicológicos (ansiedad, tristeza, irritabilidad o cambios de humor), síntomas dolorosos, síntomas gastrointestinales y síntomas circulatorios/ respiratorios (El Ansari et al., 2014). Cabe señalar que, si bien los estudiantes reportan bajo consumo de medicamentos, los más mencionados fueron los analgésicos, antidepresivos y ansiolíticos, lo que coincide con la sintomatología seńalada anteriormente.

Las quejas de salud señaladas suelen ser problemáticas agudas y raramente incapacitantes. Este resultado coincide con los altos niveles de función física (capacidad para realizar actividades básicas) del SF-36. Sin embargo, estas problemáticas si pueden estar afectando el sentido de bienestar del estudiante. A ello se agrega que los niveles de salud mental sean significativamente menores que los de salud física en este grupo, lo que podría estar relacionado con que las molestias señaladas podrían estar afectando los niveles de energía (vitalidad), y favorecer que los problemas emocionales interfieran en las actividades de los estudiantes (rol emocional).

Cabe señalar que todas las molestias señaladas son sensibles a condiciones de estrés crónico, especialmente a nivel de síntomas psicológicos; por ello, alta prevalencia de quejas psicológicas aumenta el riesgo de transformarse eventualmente en condiciones de salud más serias como sería el desarrollo de sintomatología depresiva y/o ansiosa (El Ansari et al. 2014). De hecho, esto podría estar vinculado a que en estudios con muestras universitarias se han hallado presencia de niveles clínicos de depresión con probable significado clínico entre el 15 al $50 \%$ de las muestras evaluadas y respecto a la presencia de sintomatología ansiosa se encuentran reportes entre el 15 al 40\% en grupos estudiantes evaluados (Amézquita et al., 2003; Gutiérrez et al., 2010; Pérez-Villalobos et al. 2011). 
Por otro lado, las relaciones entre los diferentes indicadores de salud y bienestar encontrados en este estudio apuntan a señalar que estos son dos conceptos ligados, pero también independientes. De forma que, es posible inferir que el deterioro de la salud, especialmente mental, de todas maneras, tendrá algún impacto en el bienestar del estudiante. Esté vinculo puede ser explicado en tanto la salud puede ir progresivamente interfiriendo en el rendimiento o en la adaptación a la vida universitaria (Chau \& Saravia, 2014), lo cual interferiría en la consecución de las metas personales centrales que son aquellas alrededor de las cuales se establece el sentido de bienestar.

Existen varios factores asociados a la salud y bienestar en estudiantes; este estudio se concentra en analizar el rol de variables sociodemográficas, académicas y conductuales reportadas por los estudiantes sin tomar en cuenta otras variables psicológicas que la investigación científica ha resaltado como la personalidad (Bobić et al., 2015; Boro \& Dhanalakshmi, 2015; Cheng et al., 2015), estrés percibido (Chau \& Saravia, 2016; David \& Brantley, 2004); estrés académico (Burris et al., 2009; Feldman et al., 2008) u otros. Si bien los modelos de regresión finales explican porcentajes pequeños de varianza de cada indicador de salud y bienestar, son significativos y aclaran el rol de estas condiciones sobre la salud y bienestar en el estudiante; además de que permite reconocer cuáles de todas las condiciones del estudiante podrían ser tomados en cuenta al momento de pensar en políticas para la promoción de la salud dentro de la universidad.

En todos los modelos de salud, se encuentra que las únicas variables académicas predictoras son el tipo de carrera y la percepción de satisfacción con el rendimiento en la universidad y para bienestar se les suma a las primeras, la percepción de satisfacción con la carrera. Llama la atención que ni la facultad a la que pertenece el estudiante, el nivel o el ciclo de estudio en el que se encuentre tiene alguna relación importante con la salud. Esto podría deberse a que, la mayoría de estudios que señalan la importancia de la facultad o el ciclo se realizaron en muestras limitadas, con pocas facultades o sin incorporar alumnos de todos los niveles de estudio; siendo por ello, que se resaltaron 
diferencias muy específicas al marco exclusivo de la investigación (Martin, 2007). Además, algunos de estos estudios incorporaron a estudiantes de carreras de medicina y veterinaria (Mazo et al., 2013), carreras que no se imparten dentro de la universidad seleccionada.

En términos de tipo de carrera se encontró que los estudiantes de carreras de ciencias mostraban mayores niveles de salud que los de carreras de letras, ello llama la atención pues dentro del imaginario colectivo sobre del nivel de exigencia de las carreras se cree que las primeras son más exigentes y demandantes y por tanto son las que más deterioran la salud. Sin embargo, es posible que sea el estilo de pensamiento más racional y orientado a la tarea de este grupo el que favorecería una aproximación menos problematizante a su situación. Estudios previos con población similar encuentra un mayor uso de algunas estrategias no productivas (como hacer ilusiones o autoinculparse) en estudiantes del área de letras mientas que en los de ciencias hay un mayor uso de estrategias productivas como distracción física (Mikkelsen, 2009; Moreano, 2009).

Sin embargo, esto podría explicarse pues en el grupo de carreras de ciencia hay una mayor proporción estudiantes varones (alrededor del 70\%) y que se dirigen o estudian carreras de ingeniería. El Ansari y Stock (2016) en un estudio con 11 facultades de una universidad de Egipto, encontraron que los estudiantes de la facultad de Ingeniería mostraban menores niveles de "conciencia de la propia salud" siendo por ello menos evidente para ellos las dificultades que pueden estar pasando, y menos probable que detecten las señales de dificultades en su salud hasta que están se desarrollen en patología más evidentes.

Respecto a las variables sociodemográficas, se encuentra diferencias importantes en los niveles de salud en función al sexo; así, se encuentra que los estudiantes varones reportan mayores niveles de salud percibida global, salud física y salud mental; lo cual coincide con investigaciones previas con universitarios (Becerra, 2013; CornelisseVermaat et al., 2006; El Ansari \& Stock, 2016; El Ansari et al. 2011; Latas et al., 2014). Algunas de estas diferencias podrían deberse a la mayor conciencia que podrían tener las mujeres sobre a propia salud 
(Idler \& Benyamin, 1997) o las prácticas de búsqueda de tratamiento y exposición a riesgo que favorece que las mujeres busquen tratamiento de manera más rápida y que los varones tengan una mayor tendencia a exposición de riesgos en salud (Buvinić et al., 2006).

Sin embargo, las variables que predicen mejor la salud en el grupo evaluado son dos conductas de salud (práctica de ejercicio físico y mantener un número de sueño adecuado) y la satisfacción con el rendimiento. Sobre la práctica de ejercicio físico, existe evidencia que el ejercicio puede ser útil para reducir el riesgo a múltiples desordenes de salud física y mental, promueve la liberación de endorfinas, produce cambios en el metabolismo de la serotonina pero también está relacionada a la promoción de un sentido de eficacia y autoestima, y a mejoras cognitivas importantes (Walsh, 2011) y por tanto, está relacionada con mejores niveles de salud percibida (Bastardo, 2011; Cheng et al, 2015; Mikolajczyk et al., 2008). En nuestra muestra, se encuentra que los beneficios de la práctica de ejercicio físico también se podrían estar dando a nivel de los diferentes indicadores de bienestar, ya que el ejercicio no solo reduce el riesgo sino que promueve emociones positivas y se considera un recurso importante para la regulación de emociones positivas proveyendo placer, vigor y energía a la persona (Woo et al., 2009).

Por otro lado, el número de horas de sueño, clasificado entre adecuado e inadecuado de acuerdo a las pautas brindadas por la OMS (Hirshkowitz et al., 2015), podría deberse a que esta práctica contribuye a una mejor calidad de sueño y por tanto, reduce la posibilidad de sufrir diversas problemáticas de salud física y mental (Taylor et al., 2003); además de contribuir al buen funcionamiento de diversos procesos cognitivos y se ha vinculado con rendimiento académico (Baert et al., 2015). En nuestro contexto se ha encontrado que la calidad de sueño se vincula con la adaptación a la vida universitaria (Pretty, 2016) y con mayores niveles de bienestar (Becerra, 2018). Por ello, se encuentra comprensible que el tipo de sueño también sea un predictor de afecto negativo en nuestra muestra.

Satisfacción con el rendimiento se relacionó de manera importante tanto con salud y bienestar y ello puede deberse al impacto que puede 
tener la percepción positiva de desempeño en la universidad con los niveles de autoeficacia general y ante las tareas académica. Así, mientas más elevada sea esta percepción mayor persistencia, dedicación y satisfacción con las acciones que realizamos (Garrido, 2000; Martínez et al., 2002) y se contaría con un recurso personal que ayudaría al estudiante a enfrentarse a situaciones demandantes (Meseguer et al., 2014). La satisfacción con la carrera, se vuelve un predictor importante en el caso de bienestar más no de la salud, en tanto se entiende que, esta provee un sentido y propósito a lo que el estudiante está haciendo, brindándole un sentido de florecimiento, afectos positivos y reduciendo la posibilidad de afectos negativos.

Finalmente, tener pareja y estar trabajando aportan de manera diferencial a los indicadores de bienestar. Los alumnos que cuentan con pareja refieren mayor afecto positivo y sentido de florecimiento que los que no cuentan con ello, siendo posible que esta condición refleja uno de los múltiples efectos positivos de contar con una red de soporte percibida además que satisfacer estos vínculos sociales se vincula con la promoción de afectos positivos (Tay \& Diener, 2011). Los alumnos que trabajan reportan menores afectos negativos, aspectos que podría deberse a que el estudiante que también trabaja tendría que focalizarse en las tareas que realiza para poder cumplirlas exitosamente, sin tener tiempo o disposición para cuestionarse o centrarse en lo negativo de la experiencia.

Si bien consideramos un aporte de este estudio el que se centre en aclarar el rol que pueden tener una serie de variables sociodemográficas y académicas sobre la salud y bienestar del estudiante; consideramos que no hace una revisión exhaustiva de todas las condiciones de este tipo, siendo esta su principal limitación. Además, este tipo de estudio no cuenta con un muestreo plenamente representativo de todas las facultades y niveles de estudio, por lo que no es posible ningún tipo de generalización, lo cual deja la posibilidad de revisar el rol de estas condiciones en universidades con una organización diferente al de la universidad seleccionada. Cabría que preguntarse de qué manera el ser estudiante de una institución pública o privada podría relacionarse con demandas distintas que se relacionarían con resultados distintos. 
Consideramos que los resultados brindados pueden ser insumos para que las universidades tomen en cuenta el cuidado de la salud en sus estudiantes. De igual forma, se podría tomar en cuenta las particularidades de las molestias de salud señaladas, y se pueden reorientar sus servicios hacia el desarrollo de programas que minimicen el impacto de las situaciones estresantes, especialmente aquellas de naturaleza académica. Más cuando, el propio Ministerio de Salud del Perú señala que las universidades son comunidades con un excelente potencial para la promoción de la salud y que es necesaria la investigación para la identificación de factores relacionados a la salud, ya que cada comunidad universitaria posee sus propias características. (Ministerio de Salud. Resolución Ministerial 968-2010).

\section{Referencias}

Agudelo, D., Casadiegos, C., \& Sánchez, S. (2008). Características de ansiedad y depresión en estudiantes universitarios. International Journal of Psychological Research, 1(1), 34-39.

American College Health Association [ACHA] (2015). American College Health Association-National College Health Assessment II: Reference Group Executive Summary Spring 2015. Hanover, MD: American College Health Association.

Amézquita, M., Gonzáles, R., \& Zuluaga, D. (2000). Prevalencia de la depresión, ansiedad y comportamiento suicida en la población estudiantil de pregrado de la Universidad de Caldas. Revista Colombiana de Psiquiatría, 32(4), 341-356.

Amigo, I., Fernández, C., \& Pérez, M. (2003). Manual de Psicología de la salud. Madrid: Pirámide.

Baert, S., Omey, E., Verhaest, D., \&Vermier, A. (2015). Mister Sandman, bring me good marks!. On the relationship between sleep quality and academic achievement. Social Science \& Medicine, 130, 91-98. https://doi.org/10.1016/j.socscimed.2015.02.011 
Barraza, A. (2003). El estrés académico en los alumnos de postgrado de la Universidad Pedagógica de Durango. En Memoria electrónica del VII Congreso Nacional de Investigación Educativa, Guadalajara, México.

Barraza, A. (2007). Propiedades psicométricas del Inventario SISCO del estrés académico. Recuperado de http://www.psicologiacientifica. $\mathrm{com} /$ sisco-propiedades-psicometricas/

Barraza, A. (2008). Características del estrés académico de los alumnos de educación media superior. México: Universidad Pedagógica de Durango. Investigación Educativa.

Bastardo, Y. (2011). Health status and health behavior in Venezuelan Pharmacy Students. Value in Health, 1(14). 122-125. https:// doi.org/10.1016/j.jval.2011.05.020

Becerra, A. (2018). Relaciones entre la calidad de sueño con el estrés académico y bienestar en universitarios (tesis de licenciatura en Psicología, con mención en Psicología Clínica). Pontificia Universidad Católica del Perú. Lima, Perú.

Becerra, S. (2013). Universidades saludables: una apuesta a una formación integral del estudiante. Revista de Psicología, 31(2), 286-314.

Bobić, J., Cvijetć, S., \& Macan, J. (2015). Personality and selfperception of physical and emotional health among first-year university students. Drustvena Istrazivanja, 24(2), 219-237. https://doi.org/10.5559/di.24.2.03

Boro, J., \& Dhanalakshmi, D. (2015). Spirituality, personality and general among college student. Indian Journal of Health and Wellbeing, 6(5), 475-479.

Buvinić, M., Medici, A., Fernández, E., \& Torres. A.C. (2006). Gender Differentials in health. En D. T. Jamison, J. G. Breman, A. R. Meashman, G. Alleyne, M. Claeson, D. B. Evans, P. Jha, A. Mills., \& P. Musgrove (Eds.), Disease Control Priorities in Developing Countries. Nueva York: Oxford University Press.

Burris, J., Brechting, E., Salsman, J., \& Carlson, Ch. (2009) Factors associated with the psychological well-being and distress of 
university students. Journal of American College Heath, 57(5), 536-543. https://doi.org/10.3200/JACH.57.5.536-544

Cassaretto, M. \& Martínez, P. (2017). Validación de las escalas de bienestar, de florecimiento y afectividad. Pensamiento Psicológico, 15(1), 19-31. https://doi.org/10.11144/Javerianacali. PPSI15-1.VEFA

Chandola, T. \& Jenkinson, C. (2000). Validating self-rated health in different ethnic groups. Ethn Health, 5(2), 151-159. https:// doi.org/10.1080/713667451

Chau, C. \& Saravia, J.C. (2014). Adaptación universitaria y su relación con la salud percibida en una muestra de jóvenes de Perú. Revista Colombiana de Psicología, 23(2), 269-284. https:// doi.org/10.15446/rcp.v23n2.41106

Chau, C. \& Vilela, P. (2016). Determinantes de la salud mental en un grupo de estudiantes de Lima y Huánuco. Revista de Psicología, 35(2), 387-422. https://doi.org/10.18800/psico.201702.001

Cheng, C-H.E., Weiss, J.W., \& Siegel, J.M. (2015). Personality traits and health behaviors as predictors of subjective wellbeing among a multiethnic sample of university-attending emerging young adults. International Journal of Wellbeing, 5(3), 21-43. https:// doi.org/10.5502/ijw.v5i3.2

Consorcio de Universidades (2006). Perfil de los estudiantes del Consorcio de Universidades: Resumen y análisis. Lima: Consorcio de Universidades.

Cornelisse-Vermaat, J., Antonides, G., van Ophem, J., \& van den Brink., H. (2006). Body Mass Index, Perceived Health, and Happiness: Their Determinants and Structural Relationships. Social Indicators Research, 79(1), 143-158. https://doi. org/10.1007/s11205-005-4097-7

Dattalo, P. (2010). Strategies to Approximate Random Sampling and Assignment. Oxford: Oxford University Press.

Diener, E., Wirtz, D., Tov, W., Kim-Prieto, C., Choi, D., Oishi, S., \& Biswas-Diener, R. (2010). New Well-Being Measures: Short Scales to Assess Flourishing and Positive and Negative 
Feelings. Social Indicators Research, 97(2), 143-156. https://doi. org/10.1007/s11205-009-9493-y

El Ansari, W., Labeed, S., Moseley, L., Kotb, S., \& El-Houfy, A. (2013). Physical and psychological well-being of university students: Survey of eleven faculties in Egypt. International Journal Preventive Medicine, 4, 293-310.

El Ansari, W., Oskrochi, R., \& Haghgoo, G. (2014). Are student's symptoms and health complaints associated with perceived stress at university? Perspectives from the United Kingdom and Egypt. International Journal of Environmental Research and Public Health, 11(10), 9981-10002. https://doi.org/10.3390/ ijerph111009981

El Ansari, W. \& Stock, C. (2016). Explaining the gender difference in self-rated health among university students in Egypt. Women \& Health, 56(7), 731-744. https://doi.org/10.1080/03630242.20 15.1118733.

El Ansari, W., Stock, C., Snelgrove, S., Hu, X., Parke, S., Davies, S., John, J., Adetunji, H., Stoate, M., Denny, P., Phillips, C., \& Mabhala, A. (2011). Feeling healthy? A survey of physical and psychological wellbeing of students from seven universities in the UK. International Journal of Environmental Research and Public Health, 8(5), 1308-1323. https://doi.org/10.3390/ ijerph8051308

Feldman, L., Goncalves, L., Chacón-Puignau, G., Zaragoza. J., Bagés, N., \& De Pablo, J. (2008). Relaciones entre estrés académico, apoyo social, salud mental y rendimiento académico en estudiantes universitarios venezolanos. Universitas Psychologica, 7(3). 739-751. Colombia: Pontificia Universidad Javeriana.

Garrido, E. (2000). Autoeficacia percibida: el origen de una teoría. Universidad de Salamanca.

González-Ramírez, M., Landero-Hernández, R., \& García-Campayo, J. (2009). Relación entre la depresión, la ansiedad y los síntomas psicosomáticos en una muestra de estudiantes universitarios del 
norte de México. Revista Panamericana de Salud Pública, 5(2). $141-145$.

Gutiérrez, J., Montoya, L., Toro. B., Briñon, M., Rosas, E., \& Salazar, L. (2010). Depresión en estudiantes universitarios y su asociación con el estrés académico. Revista CES Medicina. 24(1), 7-17. Hirshkowitz, M., Whiton, K., Albert, S., Alessi, C., Bruni, O., DonCarlos, L., Hazen, N., Hernan, J., Katz, E., KheirandishGozal, L., Neubaurer, D., O’Donnell, A., Ohayon, M., Peever, J., Rawding, R., Sachdeva, R., Setters, B., Vitiello, M., Ware, J., \& Adams, P. (2015). National Sleep Foundation's sleep time duration recommendations: methodology and results summary. Sleep Health. Journal of the National Sleep Foundation, 1(1). 40-43.

Hussain, R., Guppy, M., Robertson, S., \& Temple, E. (2013). Physical and mental health perspectives of first year undergraduate rural university students. BMC Public Health, 13, 848. https://doi. org/10.1186/1471-2458-13-848

Idler, H. \& Benyamin, Y. (1997). Self-Rated Health and Mortality: A Review of Twenty-Seven Community Studies. Journal of Health and Social Behavior, 38(1), 21-37.

Kline, R. (2010). Principles and Practice of Structural Equation Modeling (3era ed.). New York: Guilford Press.

Lange, I. \& Vio, F. (2006). Guia para Universidades Saludables y otras Instituciones de Educación Superior. Santiago: Instituto de Nutrición y Tecnología de los Alimentos y la Pontificia Universidad Católica.

Latas, M., Stojkovic, T., Ralic, T., Jovanovic, S., Spiric, Z., \& Milovanovic, S. (2014). Medical student's health related quality of life- A comparative study. Vojnosanit Pregl, 71(8), 751-756. https://doi.org/10.2298/VSP1408751L

Li, F., Bai, X. \& Wang, Y. (2013). The Scale of Positive and Negative Experience (SPANE): Psychometric Properties and Normative Data in a Large Chinese Sample. PLOS ONE. 8(4). 469-478. https://doi.org/10.1371/journal.pone.0061137 
Mazo, R., Londoño, K., \& Gutiérrez, Y. (2013). Niveles de estrés académico en estudiantes universitarios. Informes Psicológicos, 13(2), 121-134.

Martín, M. (2007). Estrés académico en estudiantes universitarios. Apuntes de Psicología, 25(1), 87-99.

Martínez, I., Marques, A., Salanova, M., \& López da Silva., A. (2002). Burnout en estudiantes universitarios de España y Portugal. Ansiedad y Estrés, 8(1), 13-23.

Meseguer, M., Soler, M., \& García-Izquierdo, M. (2014). El papel moderador de la autoeficacia ente situaciones de acoso laboral y la salud en una muestra multiocupacional. Anales de Psicología, 30(2), 573-578.

Micin, S. \& Bagladi, V. (2011). Salud mental en estudiantes universitarios: incidencia de psicopatología y antecedentes de conducta suicida en población que acude a un servicio de salud estudiantil. Terapia Psicológica, 29(1), 53-64.

Mikkelsen, F. (2009). Satisfacción con la vida y estrategias de afrontamiento en un grupo de adolescentes universitarios de Lima (tesis de licenciatura en Psicología con mención en Psicología Clínica). Pontificia Universidad Católica del Perú, Lima.

Mikolajczyk, R., Brzoska, P., Maier, C., Ottava, V., Meier, S., Dudziak, U., Ileva, S., \& El Ansari, W. (2008). Factors associated with self-rated health status in students: a cross-sectional study in three European countries. BMC Public Health, 8, 1-10.

Moreano, L. (2006). Estilos de personalidad y estrategias de afrontamiento en adolescentes universitarios (tesis de licenciatura en Psicología con mención en Psicología Clínica). Pontificia Universidad Católica del Perú, Lima.

Organización Mundial de la Salud - OMS (2002). Informe sobre la salud en el mundo. Reducir los riesgos y promover una vida sana. Recuperado de http://www.who.int/whr/2002/en/whr02_es.pdf

Pérez-Villalobos, C., Bonnefoy-Dibarrat, C., Cabrera-Flores, A., Peine-Grandón, S., Abarca, K-. Baqueano-Rodríguez, M., \& Jiménez-Espinoza, J. (2012). Problemas de salud mental en 
alumnos universitarios de primer año de Concepción, Chile. Anales de Psicología, 28(3), 797-804.

Pinquart, M. (2001). Correlates of subjective health in older adults: a meta-analysis. Psychol Aging, 16(3), 414-426. https://doi. org/10.1037/0882-7974.16.3.414

Pretty, B. (2016). Calidad de sueño y adaptación a la vida universitaria en estudiantes universitarios (tesis de licenciatura en Psicología, con mención en Psicología Clínica). Pontificia Universidad Católica del Perú, Lima.

Ryan, R. \& Deci, E. (2001). On Happiness and Human Potentials: A Review of Research and Hedonic and Eudaimonic WellBeing. Annual Review of Psychology, 52, 141-166. https://doi. org/10.1146/annurev.psych.52.1.141

Salazar, F. \& Bernabé, E. (2012). The Spanish SF-36 in Peru: Factor Structure, Construct Validity and Internal Consistency. Asia Pacific Journal of Public Health, 13, 1-9. https://doi. org/10.1177/1010539511432879.

Silva, A. \& Caetano, A. (2013). Validation of the Flourishing Scale and Scale of Positive and Negative Experience in Portugal. Social Indicators Research, 110(2), 469-478. https://doi.org/10.1007/ s11205-011-9938-y

Tay, L. \& Diener, E. (2011). Needs and Subjective Well-Being around the world. Journal of Personality and Social Psychology, 101(2), 354-365. https://doi.org/10.1037/a0023779

Taylor, S. (2007). Psicología de la salud. México: McGraw-Hill Interamericana.

Taylor, D., Lichstein, K., \& Durrance, H. (2003). Insomnia as a health risk factor. Behavioral Sleep Medicine, 1(4), 227-247. https:// doi.org/10.1207/S15402010BSM0104_5

Walsh, R. (2011). Lifestyle and Mental Health. American Psychological Association. Vol. 66 (7), 579-592

Ware, J. \& Gandek, B. (1998). Overview of the SF-36 Health Survey and the International Quality of Life Assesment (IQOLA) 
Project. Journal of Clinical Epidemiology, 51(11), 903-912. https://doi.org/10.1016/s0895-4356(98)00081-x

Ware, J. \& Sherbourne, C. (1992). The MOS 36-Item Short-Form Health Survey (SF-36): I. conceptual framework and item selection. Medical Care, 30(6), 473-483.

Ware, J., Snow, K., Kosinski, M., \& Gandek B. (1993). SF-36 Health Survey: manual and interpretation guide. Boston: New England Medical Center, the Health Institute.

Woo, M., Kim, S., Kim, J., Petruzzello, S. J., \& Hatfield, B. D. (2009). Examining the exercise-affect dose-response relationship: Does duration influence frontal EEG asymmetry? International Journal of Psychophysiology, 72(2), 166-172. https://doi.org/10.1016/j. ijpsycho.2008.12.003

Recibido: 27 de febrero, 2019

Revisado: 6 de octubre, 2019

Aceptado: 6 de enero, 2020 